
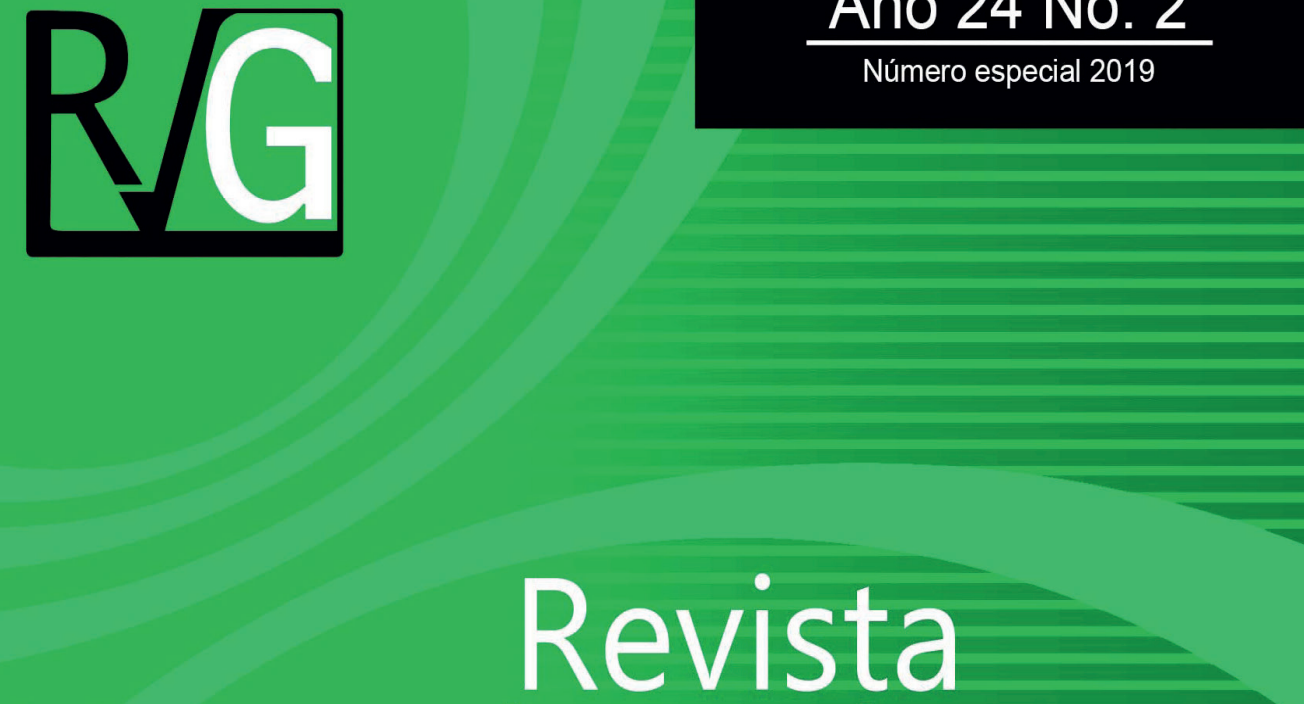

Venezolana de

$\frac{1}{6}$

$\frac{\mathbb{O}}{\frac{1}{0}}$
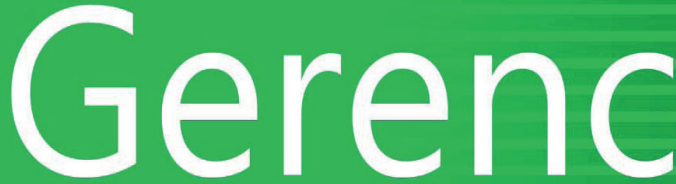

0

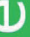

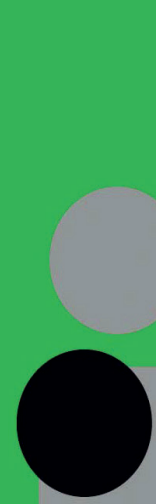

ro

u

$>$

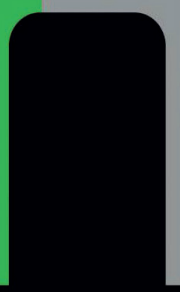




\title{
Perfil emprendedor en estudiantes: divergencias entre dos universidades colombianas
}

\author{
Rubio-Rodríguez, Gustavo Adolfo' \\ Rodríguez Barrero, Mario Samuel ${ }^{2}$ \\ Flórez Guzmán, Mario Heimer ${ }^{3}$ \\ Molina Ramírez, Adriana Rosa ${ }^{4}$
}

\section{Resumen}

El presente escrito tiene como propósito, analizar las diferencias del perfil emprendedor entre los estudiantes de administración de empresas de dos universidades colombianas. La aproximación teórica se centra en los modelos de emprendimiento y su medición. El enfoque de la investigación es mixto y de alcance descriptivo; las unidades muéstrales seleccionadas en las universidades objeto de estudio corresponde a 196 y 173, respectivamente. El instrumento de recolección de información, está basado en la metodología GEM y fue validado por la Red de Investigadores de Ascolfa. Los resultados presentan las convergencias y divergencias encontradas entre el perfil de los estudiantes de las instituciones analizadas. Como conclusión, al adelantar el análisis comparativo en las dos universidades, se observó que coexiste una influencia del contexto social y económico en las ciudades donde éstas operan, las cuales presentan procesos urbanos de crecimiento y desarrollo en los últimos años.

Palabras clave: emprendimiento; administración de empresas; perfil emprendedor; instituciones de educación superior.

\section{Recibido: Aceptado:}

1 Doctor of Management with a major in Management and Administration Project, Doctor en Ciencias Económicas y Administrativas, Magister en Administración de Empresas, Master MBA en Dirección Empresarial y Marketing. Profesor investigador Programa de Contaduría Pública, Corporación Universitaria Minuto de Dios. Correo: gustavo.rubio-r@uniminuto.edu.co

2 Doctorando en Administración Gerencial, Magíster en Dirección de Marketing, Especialista en Gerencia de mercadeo, Administrador de empresas, Investigador asociado en Colciencias, Profesor investigador Programa de Administración de Empresas, Universidad Cooperativa de Colombia. Correo: mario. rodriguezb@campusucc.edu.co

3 Doctor en Ciencias de la Educación, Profesor Corporación Universitaria Remington. Licenciado en matemáticas y física, con estudios de doctorado en educación. Experto en responsabilidad social corporativa y auditoría forense. Profesor universitario categorizado en senior por el ministerio colombiano de ciencia, tecnología e innovación. Centro de Investigaciones e Innovación en los Negocios CIIN Colombia. Correo: mariohfg@hotmail.com.

4 Magister en Educación. Profesora Corporación Universitaria Remington. Correo: adriana.molina@ uniremington.edu.co. ORDCID: 0000-0001-9499-9166. 


\title{
Entrepreneurial profile in students: divergences between two Colombian universities
}

\begin{abstract}
The purpose of this document is to analyze the differences in the entrepreneurial profile between the Business Administration students of two Colombian universities in the city of Ibagué, Colombia. The theoretical approach focuses on entrepreneurship models and their measurement. The research approach is mixed and descriptive in scope; the sample units selected in the universities under study correspond to 196 and 173, respectively. The information collection instrument is based on the GEM methodology and was validated by the Ascolfa Researchers Network. The results present the convergences and divergences found among the profile of the students of the analyzed institutions. In conclusion, when advancing the comparative analysis in the two universities, it was observed that an influence of the social and economic context coexists in the cities where they operate, which present urban processes of growth and development in recent years.
\end{abstract}

Keywords: Business administration; entrepreneurship; entrepreneurial profile; Higher education institutions

\section{Introducción}

Según el Departamento Administrativo Nacional de Estadística -DANE- (2019), para el año 2018 el porcentaje de personas en situación de pobreza en Colombia fue del $27 \%$, el de personas en situación de pobreza extrema $7,2 \%$ y el coeficiente de Gini registró 0.517 ; estas cifras evidencian que Colombia sigue siendo uno de los que países en donde se presenta mayor desigualdad y pobreza de la región, resaltando que la mayor incidencia de pobreza extrema está asociada entre otros factores, con el nivel educativo.

En escenarios como estos, el emprendimiento tiene un papel muy importante, pues a través de la actividad emprendedora, se crean empresas, se genera empleo, se disminuye la pobreza, y la desigualdad, además, es posible mejorar la calidad de vida de los ciudadanos (Duran, Fuenmayor, Cárdenas, y Hernández, 2016). Entendiendo esto, la promoción del emprendimiento se ha convertido en un reto para el Estado y en especial para sistema educativo colombiano, pues es a través de los emprendedores es que se crean empresas y se generan nuevas oportunidades de empleo y progreso.

Por este motivo, el Gobierno Nacional ha buscado estimular la formación en emprendimiento, trazando una política nacional en torno a este tema, para impulsar el nacimiento y crecimiento de las empresas, generar desarrollo económico y disminuir la desigualdad (Ley 1014, 2006), pues desde esta 
Perfil emprendedor en estudiantes: divergencias entre dos universidades colombianas Rubio-Rodríguez, Gustavo Adolfo; Rodríguez Barrero, Mario Samuel;

Flórez Guzmán, Mario Heimer y Molina Ramírez, Adriana Rosa

Ley se entiende el emprendimiento como "una forma de pensar, razonar y actuar, centrada en las oportunidades, planteada con visión global y llevada a cabo mediante la gestión de un riesgo calculado; su resultado, es la creación de valor que beneficia a la empresa" (Articulo $1^{\circ}$ Definiciones).

En cuanto a la formación en emprendimiento, las universidades tienen un papel determinante, pues durante la etapa de formación académica, los estudiantes pueden desarrollar una cultura emprendedora, y esto se logra, si la universidad incorpora en su currículo, estrategias a través de las cuales se incentive y fomente el emprendimiento, con actividades de formación, desarrollo de competencias, ambientes empresariales, y creando todo un clima que motive a los estudiantes a emprender, pues la universidad es el campo propicio para desarrollar actividades de emprendimiento efectivo, que trasciendan en el tiempo (Ruiz, Cabeza, y Briano, 2012; Durán, Parra, y Márceles, 2015).

En este contexto se enmarca la investigación que fundamenta este artículo, pues a través del estudio realizado, se buscó generar respuesta a algunas de las cuestiones anteriormente mencionadas, a partir de una revisión de fuentes secundarias $y$ fuentes primarias, tanto a nivel nacional como regional, teniendo en cuenta las sedes universitarias estudiadas. EI planteamiento del problema se enmarca inicialmente en la pregunta: ¿Cuál es el perfil emprendedor de los estudiantes de administración de empresas de dos instituciones de educación superior colombianas?

A partir de esta pregunta de investigación, se pretendía analizar las diferencias del perfil emprendedor de los estudiantes del programa de administración de empresas de dos instituciones de educación superior, ya que es interesante caracterizar este perfil en los estudiantes. Finalmente, la realización de la investigación permitirá proponer nuevas metodologías y estrategias en cada sede para incentivar y motivar la formación en emprendimiento a los estudiantes.

Como se puede evidenciar, la presente investigación aborda una problemática relevante para las universidades, en el sentido de que no se conocen cuáles son las características que identifican a un estudiante emprendedor. Para el caso de esta investigación, se tomaron como poblaciones objeto de estudio a los estudiantes del programa de administración de empresas de dos universidades, dado que tanto las estructuras curriculares de dichos programas, como las ciudades donde operan, poseen características similares, por lo cual los resultados permitirán generar estrategias para fomentar el emprendimiento en los estudiantes de estas ciudades, y servirá como referente para el emprendimiento universitario a nivel nacional.

Este trabajo investigativo se adelantó bajo un enfoque mixto, tratando de analizar diferentes percepciones de los estudiantes, en cuanto a su perfil emprendedor. Bajo esta concepción, fue necesario indagar a través de entrevistas y encuetas la representación que caracteriza la población objeto de estudio, a fin de conocer sus diferentes conductas, cuyos resultados lograron reflejar los fines esperados en cada uno de los objetivos específicos, para así facilitar el cumplimiento del propósito general. 


\section{Evolución del concepto de emprendimiento}

Acudiendo a fuentes comúnmente aceptadas como la Real Academia Española -RAE-, se puede considerar que el término emprender se deriva del latín in (en) y prendĕre (coger) (Diccionario de autoridades, 1732), por otra parte, Grebel, Pika, y Hanusch (2003), Filion (2003) y Kruger (2004) consideran que este término está relacionado con el vocablo francés entrepreneur, con el cual se definía a las personas que llevaban a cabo actividades militares, el cual se amplió a las personas encargadas de realizar obras de construcción, manufactura o cualquier otra $y$, aunque en este concepto inicialmente no consideraba el riesgo, este elemento fue incorporado posteriormente al concepto de emprendimiento.

Se reconoce, que la evolución del concepto de emprendimiento inicia con Cantillon (1755), quien dio al emprendimiento un enfoque económico, al considerar que los agentes económicos están clasificados en tres grupos: propietarios, prestamistas y emprendedores, siendo este último un agente que compra medios de producción y toma decisiones económicas en condiciones de incertidumbre; es así, que Valera-Loza, y Vara-Horna (2007), siguiendo el modelo de Cantillon, define al emprendedor como un agente que compra una materia prima a un precio cierto para transformarla y venderla a un precio incierto, asumiendo el riesgo, en la búsqueda de una oportunidad para generar utilidades que le beneficien.

Por su parte Say, en su Traité d'économie politique, presenta al emprendedor, como la persona que dirige una empresa, en el marco de un contrato, actuando como intermediario entre el capital y el trabajo (1803). Para este autor, el emprendedor debe enfrentar los riesgos y aprovechar de todo lo que puede serle favorable, es decir, las oportunidades, un concepto que hoy en día, se relaciona directamente con la gerencia estratégica. Uno de sus aportes al emprendimiento fue el de aclarar la diferencia entre el agente capitalista y el emprendedor, donde este último se considera un agente de cambio. En este sentido, Grebel et. al (2003), considera que el emprendedor es un agente activo que permite la conexión entre la oferta y la demanda.

De igual forma, Joseph Schumpeter (1911) sugiere que las invenciones e innovaciones son la clave del crecimiento económico y considera que quienes implementan ese cambio son los emprendedores; para Schumpeter la clave del emprendimiento está en la capacidad del emprendedor para transformar innovaciones en productos prácticos que hagan la vida más fácil, asumiendo un riesgo económico, al ponerlo en un mercado altamente competitivo; de esta manera, Schumpeter asoció la innovación al concepto de emprendimiento, aduciendo que el emprendedor es un agente de cambio económico, dada su capacidad para originar desequilibrios temporales en el mercado a través de la innovación, generando cambios en las actividades económicas. Desde estos aportes, el concepto del emprendedor evoluciona, pasando de ser un tomador de riesgos económicos a un innovador, generador de desequilibrio y cambios en los mercados (Pico, Del Rio, Trujillo, y Rodríguez, 2017).

En estudios recientes como el de Freire y Nielsen (2011), se considera que para determinar el perfil del emprendedor 
Perfil emprendedor en estudiantes: divergencias entre dos universidades colombianas Rubio-Rodríguez, Gustavo Adolfo; Rodríguez Barrero, Mario Samuel;

Flórez Guzmán, Mario Heimer y Molina Ramírez, Adriana Rosa

se deben tener en cuenta tres conjuntos de características: sus deseos y actitudes, los factores de personalidad, y los conocimientos técnicos. Con relación al perfil psicológico del emprendedor, se puede decir que es un individuo visionario y ejecutor de sus ideas, pues en su papel de visionario, puede ver oportunidades que otros no ven, y es capaz de transformar esa visión en un hecho concreto o en una solución práctica, frente un problema complejo.

El emprendedor es una persona con habilidades orientadas a la creación, la invención, el descubrimiento y la innovación. Entendida esta innovación, como la que transforma bienes, servicios o procesos, creando nuevos mercados o ampliando los existentes, y generando valor para los clientes y la empresa, aunque dicho valor no siempre es económico, pues existe una clase de emprendedor social, que crea otro tipo de valores, pero al final, sea el emprendedor lucrativo o no, siempre genera valor agregado por el sólo acto de su actividad innovadora. Por otra parte, la actividad emprendedora con fines de lucro o emprendimiento económico se puede definir como la combinación de factores productivos para el desarrollo de un proceso que transformará determinados productos, generando valor para los clientes y dejando un margen de utilidad (Selamé, 1999).

Se puede concluir entonces, que el perfil emprendedor de una persona que se caracteriza por su capacidad de encontrar soluciones sencillas a problemas complejos, por su aptitud para encontrar recursos destinados a la creación, invención, descubrimiento o innovación. El emprendedor es capaz de crear nuevas formas de solucionar problemas, de forma creativa, en un entorno específico. En la actualidad, existen diferentes modelos de emprendimiento los cuales se basan en estudiar sus procesos y las características sobre la personalidad y el entorno en que se desarrolla el emprendedor, teniendo en cuenta las variables endógenas y exógenas del ecosistema emprendedor. Dentro de los enfoques para el estudio del emprendedor se encuentran el psicológico y el sociológico.

El enfoque psicológico establece que los emprendedores son agentes con características innatas o adquiridas en los primeros años de vida diferentes a las del resto de las personas y trabaja sobre el supuesto que el emprendedor "nace y no se hace", este postulado es respaldado por McClelland (1960) y Hagen (1962), quienes consideran que el emprendedor tiene una personalidad creativa movida por una alta necesidad de logro. Por otra parte, está enfoque sociológico, según el cual las características del emprendedor son adquiridas a lo largo de su vida, mediante un proceso de socialización, donde interactúan una serie de factores económicos, sociológicos, psicológicos, culturales, y otras variables exógenas.

Desde esta perspectiva, se puede considerar que el emprendimiento está influenciado por vivencias personales, origen familiar, educación, experiencia ocupacional, estilo de vida, estructura de clase, el entorno, la cultura empresarial, entre otros factores que estimulan o no, el emprendimiento (O'Farrel, 1986). Se puede considerar que estos enfoques no son excluyentes, sino complementarios y que interactúan simultáneamente en cierto perfil de emprendedores, por lo cual, modelos como el Global Entrepreneurship Monitor (GEM), identifican tanto los aspectos psicológicos como sociológicos en la medición del emprendimiento (Torres, 2010; Bargsted, 2013). 
En este sentido, se puede concluir que existen múltiples caracterizaciones que pueden determinar el perfil del emprendedor, pero hay coincidencia en que el emprendedor es innovador, aunque no necesariamente inventor, tiene iniciativa y capacidad para poner en el mercado productos que pueden mejorar la calidad de vida y modificar el equilibrio económico. Se puede encontrar en cualquier nivel de la organización formal e informal de las empresas, con o sin ánimo de lucro, y, el entorno en el cual convive tiene una influencia positiva o negativa sobre la actividad emprendedora, tal como se puede evidenciar en los resultados del estudio GEM, los cuales se analizan más adelante.

Por otra parte, Alcaraz (2011), señala que el emprendimiento requiere de unas características en el individuo que lo identifiquen y lo hagan propenso a promover actividades e ideas que permitan su crecimiento individual y por supuesto local, regional y nacional. En ese sentido, temas como el compromiso, la perseverancia, la determinación, la capacidad de alcanzar metas, la confianza y el control, son preponderantes en la conformación de una persona emprendedora y con altas posibilidades de éxito en el mundo del siglo XXI.

De igual manera, el autor, destaca en el documento citado, que existen cinco tipos de personalidades del emprendedor, a saber:

- El emprendedor administrativo. (Usa la investigación)

- El emprendedor oportunista. (Busca oportunidades de ideas para beneficio propio)

- El emprendedor adquisitivo. (Continua innovación)

- El emprendedor incubador. (Afán por crecer y buscar oportunidades)

- El emprendedor imitador. (Genera innovación con base en lo ya existente)

Como elementos del proceso enseñanza-aprendizaje, basado en la formación por competencias se encuentran: Cualquier cambio que se quiera hacer en la evaluación del aprendizaje, debe iniciar con una visión de la situación que vive cada uno de los actores que conforman la comunidad académica. El diseño de una propuesta educativa centrada en la formación por competencias dirigida a la formación de un profesional más ético, crítico y generador de cambio en el medio, haciendo referencia al emprendimiento como característica esencial del egresado de una institución educativa.

\section{EI modelo GEM para la medición del emprendimiento}

Como se evidenció en el punto anterior, el estudio del emprendimiento no es un tema nuevo, sino, que ha sido abordado desde muchos siglos atrás, desde diferentes enfoques y culturas, sin embargo, generalmente se consideraba que el emprendimiento debía ser considerado desde la creación de las empresas y no desde antes, pero desde la aparición del proyecto Global Entrepreneurship Monitor (GEM) en 1997, esta percepción ha evolucionado, se ha perfeccionado y se ha ampliado, al considerar, no solo las características de los emprendedores, sino, su entorno, los procesos asociados al emprendimiento, y el ecosistema emprendedor, entre otros aspectos, que influyen incluso mucho antes de que entre en funcionamiento una unidad de negocio (Varela, Gómez, Vesga, y Pereira, 2014).

En el Global Entrepreneurship 
Perfil emprendedor en estudiantes: divergencias entre dos universidades colombianas Rubio-Rodríguez, Gustavo Adolfo; Rodríguez Barrero, Mario Samuel;

Flórez Guzmán, Mario Heimer y Molina Ramírez, Adriana Rosa

Monitor (2010), Kelley, Singer, y Herrington, (2016), presentan los resultados de la XVII medición mundial, en la cual se identifican características, motivaciones y ambiciones de los empresarios, así como las actitudes que las sociedades tienen hacia esta actividad y la calidad de los ecosistemas empresariales en diferentes economías que participaron de esta medición. Asimismo, en este reporte se presenta el modelo el Modelo de las fases empresariales y de emprendimiento representadas en GEM, el cual se presenta en el diagrama 1.

\section{Diagrama 1 \\ Modelo de fases empresariales y características de emprendimiento - GEM}

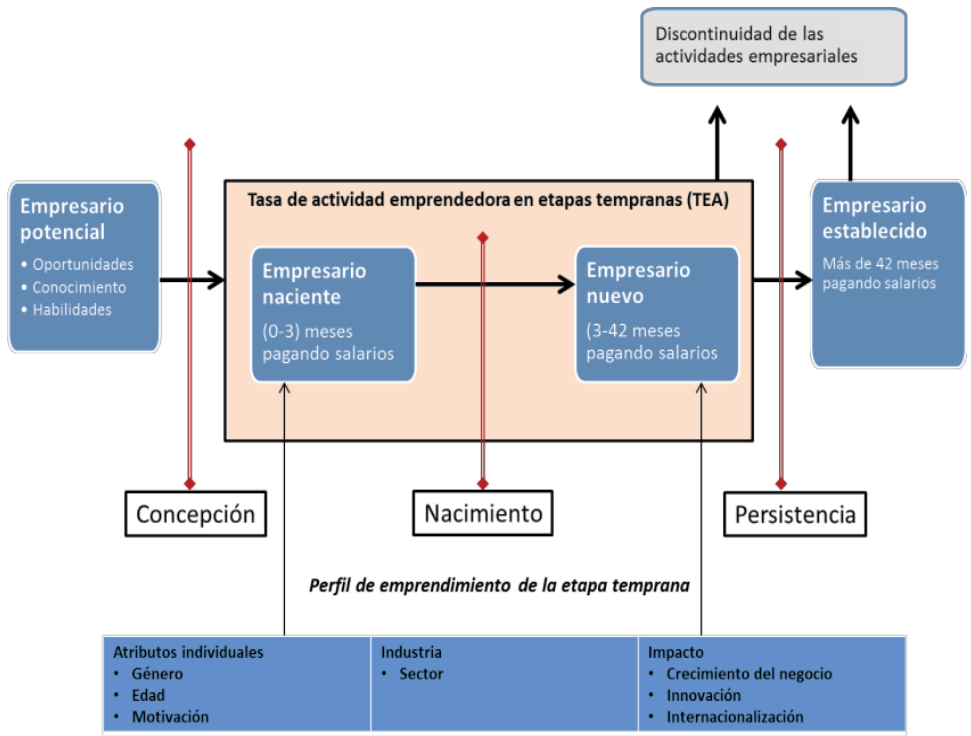

Fuente: Global Entrepreneurship Monitor (2010) Traducido por los autores\}

En este modelo, se puede observar una concepción más amplia del emprendimiento, la cual abarca desde el empresario potencial hasta el empresario establecido, considerando además elementos tanto internos como externos del emprendedor, que influyen en las diferentes etapas del proceso emprendedor. También se observa, que el proceso puede ser interrumpido en el momento de la concepción de una idea, el nacimiento de una empresa o durante el paso de la etapa temprana a la de empresario establecido.

Para ilustrar el emprendimiento efectivo y las pérdidas que se van 
presentando durante el proceso, Varela, y Soler (2012), desarrollaron el concepto de tubería empresarial, basado en la mecánica de fluidos, usando el principio de balance de la materia, para identificar las fugas que se presentan durante el proceso de emprendimiento, en las etapas de aceptación sociocultural sobre el espíritu empresarial, empresarios potenciales, empresarios intencionales, empresarios nacientes, nuevos empresarios y empresarios establecidos.

En un estudio comparativo realizado en diferentes países, por los autores Varela y Soler (2015) durante los años 2011 y 2012, se observaron los resultados para Colombia, que se presentan en el diagrama 2. Los resultados corresponden a la medición realizada durante cada etapa. La variable de la Etapa 1. Aceptación social del proceso empresarial, se mide, de acuerdo a la metodología GEM, con las siguientes preguntas: ¿considera usted que en su país acometer una nueva iniciativa empresarial es una buena selección de carrera?, ¿considera usted que en su país la sociedad otorga a los empresarios un nivel alto de status social? y, ¿considera usted que en su país, los medios otorgan relevancia positiva a los empresarios y a las iniciativas empresariales exitosas?

Para la Etapa 2. Empresarios potenciales, el modelo GEM propone las siguientes preguntas: ¿tiene usted el conocimiento, la habilidad y la experiencia necesaria para iniciar una nueva empresa?, ¿percibe usted la existencia en su entorno de oportunidades empresariales en los próximos 6 meses? y, ¿el temor al fracaso lo frenaría a usted de acometer iniciativas empresariales? Para la Etapa 3. Empresario con intención empresarial, la pregunta propuesta para la medición es: ¿está usted planeando iniciar una nueva empresa, solo o en asocio de otras personas, en los próximos tres años?

Para la medición de la actividad emprendedora en la Etapa 4. Empresario naciente, se tienen en cuenta los empresarios que durante los últimos 12 meses han realizado acciones orientadas a la creación de una nueva empresa. Para la Etapa 5. Nuevo empresario, se tienen en cuenta los empresarios que han generado ingresos o salarios por un lapso entre 3 meses y 42 meses. Finalmente, para la Etapa 6. Empresarios establecidos, se tienen en cuenta aquellos que han superado los 42 meses, generando ingresos o salarios para sus empleados. Los resultados de estas mediciones para Colombia, se presentan en el diagrama 2.

De acuerdo a esta medición, se puede concluir que Colombia presenta indicadores de aceptación social bastante altos, los cuales corresponden a la primera etapa del proceso de emprendimiento; para la siguiente etapa, que corresponde a los empresarios potenciales, se observa una caída significativa, relacionada con la percepción que tiene la población sobre los conocimientos, las habilidades y las experiencias para iniciar una empresa y el temor al fracaso.

En el presente artículo, se presta especial atención a la fuga ocasionada por la percepción que tienen los encuestados sobre los conocimientos y habilidades para el emprendimiento, pues ésta se relaciona con los procesos de formación profesional $y$, precisamente, los resultados que se presentan en este manuscrito, corresponde a la medición adelantada 


\section{Diagrama 2 Tubería Empresarial Colombia 2011-2012}

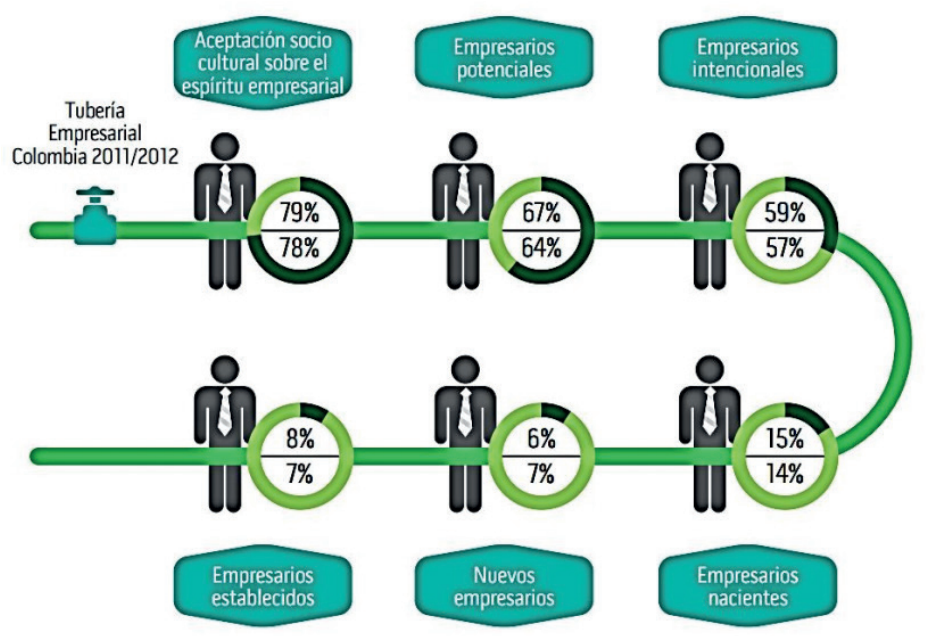

Fuente: Varela y Soler, 2015:187.

a los estudiantes del programa de administración de empresas de dos universidades, a quienes se les aplicó un instrumento, de acuerdo al Modelo GEM. Resulta entonces importante analizar dichos resultados y compararlos con los resultados nacionales.

Con relación a esta fuga, los autores recomiendan mejorar los procesos educativos, para que los conocimientos y las competencias empresariales mejoren. Proponen también reforzar los procesos de experiencias empresariales para mejorar estos indicadores. De igual forma, para evitar las fugas que se presentan en cada etapa del proceso, se proponen diversas estrategias, como la implementación de políticas y demás acciones conducentes a mantener una alta tasa de actividad empresarial -TEA-.

De otra parte, la justificación en la escogencia del modelo GEM para ser tratado en el estudio, radica en que éste analiza la propensión hacia el emprendimiento, identifica las condiciones del entorno relacionadas con la actividad emprendedora, mide la ejecución de iniciativas emprendedoras, compara la actividad empresarial de diferentes países y orienta a los gobiernos en la formulación de políticas para fomentar el emprendimiento.

Finalmente, de acuerdo a los resultados del Reporte GEM 20152016 con relación a la autopercepción sobre el emprendimiento, el índice de oportunidades percibidas en el país es de 58.3; el índice de capacidades percibidas es del 59.5; el de miedo al fracaso de 33.2 y el de intenciones empresariales de 48.2, lo cual ubica a Colombia en los primeros lugares 
con relación a la percepción sobre el emprendimiento (Kelley, Singer, y Herrington, 2016). Estos resultados se podrán comparar con los obtenidos en la medición realizada a los estudiantes universitarios.

\section{Caracterización de las universidades estudiadas e influencia de su entorno}

Bajo este contexto, las dos universidades objeto de estudio, las cuales operan como sedes de una misma universidad, tienen un gran reto con relación a la formación en emprendimiento, pues es una de las universidades más grande del país, y tiene presencia en zonas apartadas donde se acentúan los problemas sociales y atiende a población de estratos medio y bajo, para quienes el emprendimiento puede ser la mejor alternativa. Teniendo en cuenta sus ejes estratégicos transversales, las dos universidades se han trazado metas para 2022 en acreditación, docencia, investigación, extensión y proyección social, las cuales se deben enmarcar en el emprendimiento, para hacer de la universidad un ambiente propicio en la cual los estudiantes se sientan motivados para emprender.

De esta manera, ejes estratégicos como la docencia y la investigación pueden vincular el emprendimiento a través de la generación de competencias investigativas, actividades en el aula de clase que inquieten a los estudiantes a solucionar problemas y necesidades de su entorno a través de ideas reales que se puedan ejecutar, así como proyectos emprendedores, actividades extracurriculares, prácticas empresariales $y$, como ya se tiene estipulado en la normatividad, tener una opción de grado para emprendedores que, en este caso, es el plan de negocios.

En el eje estratégico de extensión y proyección social, el emprendimiento también tiene una función importante, pues se considera la extensión como la generación, difusión y transferencia de conocimiento de la universidad, a la sociedad. En las últimas décadas, se han desarrollado varios modelos que explican la relación UniversidadEmpresas-Estado, en un enfoque que pone a la universidad en función de satisfacer las necesidades de las empresas para percibir beneficios económicos por sus servicios, como por ejemplo las consultorías, asesorías, y alianzas estratégicas, con empresas públicas y privadas (Ortiz y Morales, 2011), pero la función de la universidad puede ir más allá de proveer servicios, pues a través del emprendimiento puede generar nuevas ideas de negocios, nuevas empresas, nuevas oportunidades de empleo y dinamizar la economía con innovación y desarrollo de nuevas tecnologías.

De esta manera, la universidad puede articular el emprendimiento en cada uno de sus ejes estratégicos, entendiendo el emprendimiento como un fenómeno multidimensional $y$ complejo, que se construye y prioriza de acuerdo a las concepciones, a la misión y a los intereses institucionales. En este proceso, también es importante la interacción de la universidad con sus stakeholders, pues es la interacción efectiva con el medio y el entendimiento de las necesidades del entorno, lo que permite a la universidad aproximar a los estudiantes a soluciones antes los problemas reales de la sociedad, generando un verdadero emprendimiento universitario y cumpliendo así con su 
Perfil emprendedor en estudiantes: divergencias entre dos universidades colombianas Rubio-Rodríguez, Gustavo Adolfo; Rodríguez Barrero, Mario Samuel;

Flórez Guzmán, Mario Heimer y Molina Ramírez, Adriana Rosa

responsabilidad con la sociedad (Gaete, 2017).

En el plan estratégico nacional de las universidades estudiadas, se evidencia el interés por fortalecer la formación de los estudiantes en emprendimiento, por lo cual, la vicerrectoría de proyección institucional, se ha dedicado en forma exclusiva y permanente a educación, investigación, emprendimiento solidario e innovación social, ofreciendo a los estudiantes, profesores, egresados y empleados, cursos y cátedras institucionales en forma presencial y virtual para fomentar y apoyar el emprendimiento solidario a nivel nacional e internacional, con sensibilización, apalancamiento técnico y social para la autogestión en las organizaciones solidarias, asesoría, consultoría y servicios tecnológicos para la innovación social.

\section{Diseño Metodológico}

La metodología atiende a un enfoque mixto, entendiendo el emprendimiento como un atributo humano, susceptible de medición a través de técnicas cualitativas y cuantitativas; por lo tanto, su análisis se hace a través de dichas técnicas, sin desconocer que las mediciones se presentan en términos porcentuales (cuantitativos), al igual que las comparaciones, para facilitar su comprensión y análisis.

Las unidades de análisis las constituyen los estudiantes de los programas de administración de empresas de las dos universidades estudiadas. Para el desarrollo de la investigación, los equipos de investigación de cada sede participante del proyecto, se encargaron de administrar un instrumento a las unidades muéstrales conformadas por los estudiantes de administración de empresas de las dos sedes (universidades), la cual correspondió a 398 estudiantes de la sede 1 (Ibagué) y 314 de la sede 2 (Bucaramanga).

Para el proceso de recolección de los datos, es de resaltar, que en primer lugar se realizó una búsqueda de información secundaria para tener un acercamiento al perfil emprendedor de los estudiantes universitarios, teniendo en cuenta el estado del tema. También se tuvieron en cuenta como parte de la información secundaria, los resultados obtenidos en estudios recientes sobre emprendimiento en otras universidades del oriente colombiano y los resultados del GEM durante los últimos años.

Una vez finalizada la administración de las encuestas, se procedió a la tabulación y el análisis de las variables. Adicionalmente, se aplicó la técnica de grupos focales con el fin de ampliar la información obtenida, profundizar en los aspectos relevantes y llegar a conclusiones y recomendaciones que permitieran proponer estrategias metodológicas para fomentar el emprendimiento en los estudiantes universitarios.

\section{Medición del perfil empren- dedor}

Como se presentó en el marco teórico, la metodología GEM permite medir el emprendimiento de manera amplia y consistente a través de diferentes herramientas, entre las cuales presenta indicadores percepciones que fueron tenidos en cuenta al consultar a los estudiantes del programa de administración de empresas de las dos universidades objeto de estudio; no obstante, es importante tener un contexto previo de las dos ciudades 
donde operan las universidades, para conocer la realidad en la que viven los estudiantes, lo cual puede incidir en los resultados observados.

Para el desarrollo de este proceso investigativo, se analizaron los indicadores de percepción de oportunidades, de capacidades, temor al fracaso, empresarios intencionales, el emprendimiento como carrera deseable y alto status para emprendedores, siguiendo la metodología GEM, descrita en el marco teórico. Con relación al indicador de percepción de oportunidades, el cual corresponde a las personas entre los 18 y los 64 años que identifican oportunidades para crear empresa, se consultó a los estudiantes si habían percibido oportunidades de negocio en los últimos 6 meses para la creación de nuevas empresas. Los encuestados tenían 3 alternativas de respuesta: nunca, a menudo $\mathrm{y}$, casi siempre. Según lo observado, en la sede número 1 (Ibagué), el $13 \%$ no percibió oportunidades en el último semestre, mientras en la sede número 2 (Bucaramanga) esta cifra fue del 6\%, por el contrario, el $51 \%$ de los encuestados en la sede número 2, manifestaron haber percibido oportunidades de negocios en los últimos seis meses, mientras en la sede número 1 solo el $40 \%$ de los estudiantes universitarios, declaró haber percibido este tipo de oportunidades. Los resultados a esta pregunta se presentan en el gráfico 1.

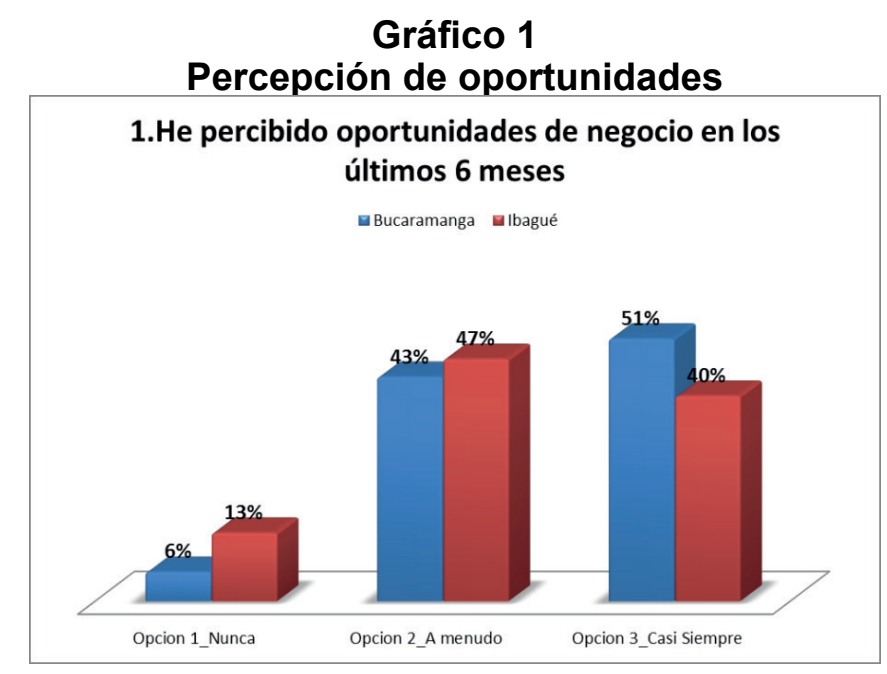

Fuente: elaboración propia

Como se puede observar en los resultados, los estudiantes de la sede número 2 tienen una mayor percepción de oportunidades para iniciar nuevos negocios que los estudiantes de administración de empresas de la sede número 1; es importante anotar, que aun cuando las condiciones del entorno 
Perfil emprendedor en estudiantes: divergencias entre dos universidades colombianas Rubio-Rodríguez, Gustavo Adolfo; Rodríguez Barrero, Mario Samuel;

Flórez Guzmán, Mario Heimer y Molina Ramírez, Adriana Rosa

fueran similares, estas percepciones pueden ser diferentes, pues obedecen a múltiples factores, como la formación, cultura, experiencias, ingresos y entorno familiar entre otros. Asimismo, al contrastar estos resultados con las realidades de cada ciudad descritas al inicio de este análisis, se puede observar mayor percepción de oportunidad en la ciudad donde hay más empleo (sede número 2), mientras que la ciudad donde reside la sede número 1 , ven menos oportunidades de negocios, precisamente donde hay mayor índice de desempleo.

En segundo lugar, se analiza la percepción de capacidades por parte de los estudiantes, para determinar si consideran que tienen las habilidades y los conocimientos requeridos para comenzar una empresa. Esta pregunta hace parte de la metodología GEM para la medición del emprendimiento, pero en este estudio tiene especial interés, dado que la población objeto de estudio corresponde a jóvenes universitarios del programa de administración de empresas; una carrera, que por definición prepara a los estudiantes para administrar unidades de negocio. Con relación a esta pregunta se obtuvieron los siguientes resultados (gráfico 2).

\section{Gráfico 2}

Percepción de capacidades

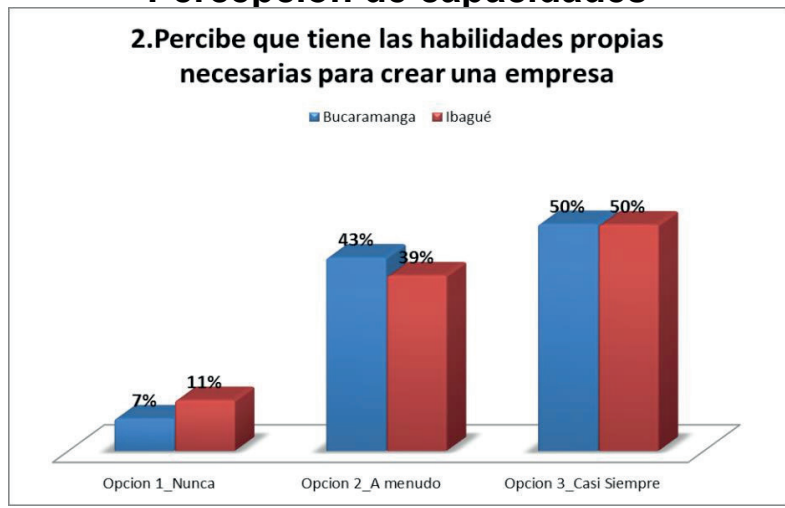

Fuente: elaboración propia

Este indicador muestra cifras claves para la universidad, pues es un mensaje de que los estudiantes no se sienten preparados para asumir el reto de crear una empresa; es también una oportunidad para que se integren al currículo, actividades donde los estudiantes creen sus propias empresas, las administren, se enfrenten al entorno y se sientan mejor preparados para crear empresa al culminar su formación. Estas respuestas fueron similares en las dos ciudades, lo cual puede obedecer a que se recibe formación similar en las dos sedes.

El tercer indicador está relacionado 
con el posible temor al fracaso. Para medir este indicador se les preguntó a los estudiantes, si consideraban que el miedo al fracaso sería un obstáculo para crear empresa. Este indicador, aunque puede estar influenciado por la educación universitaria, puede tener mayor influencia por el entorno económico, familiar, legal, los antecedentes, lo social, entre otros. Puede también ser intrínseco, es decir, que puede ser una predisposición espontánea de aversión o propensión al riesgo, un rasgo psicológico difícilmente modificable, gráfico 3

\section{Gráfico 3 \\ Temor al fracaso}

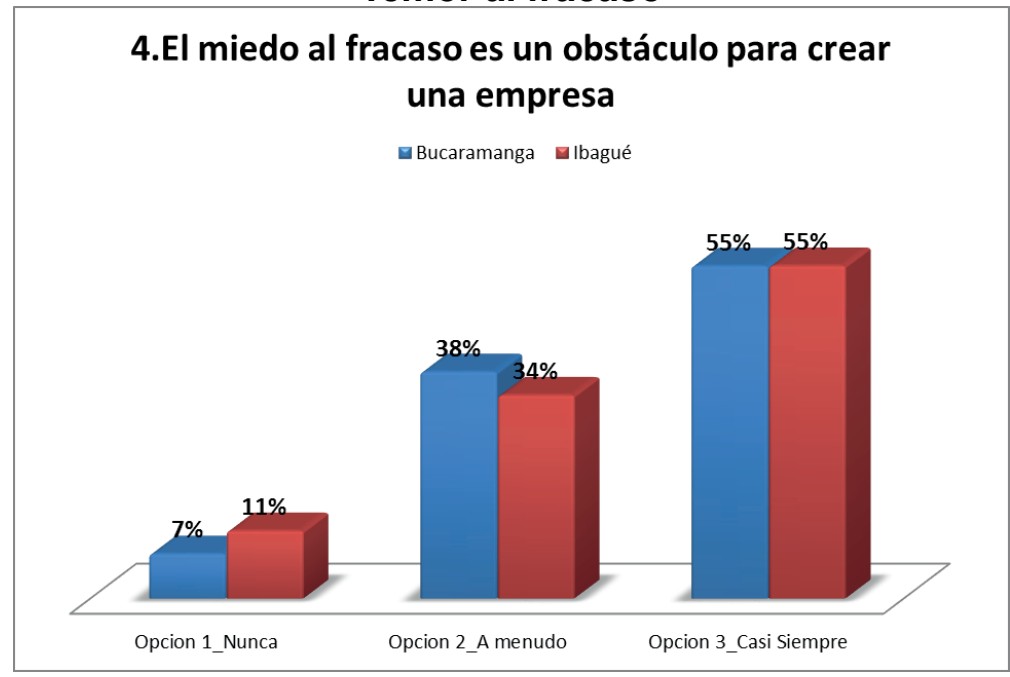

Fuente: elaboración propia

Se logró observar que la mayoría de los estudiantes manifiestan que el miedo al fracaso es un obstáculo para crear empresa; estas respuestas pueden tener dos interpretaciones, la primera que los estudiantes consideran que el miedo constituye un obstáculo para que cualquier persona cree una empresa, y otra, que los estudiantes consideran que el miedo a fracasar será un obstáculo para que ellos creen sus propias empresas; de cualquier manera, es también una oportunidad para que la universidad genere estrategias curriculares para que los estudiantes pierdan el miedo al fracaso y emprendan la creación de empresa.

Con relación al índice de los empresarios intencionales, es decir, el porcentaje de personas que no están involucrados en procesos empresariales, pero que intentarían comenzar una 
Perfil emprendedor en estudiantes: divergencias entre dos universidades colombianas Rubio-Rodríguez, Gustavo Adolfo; Rodríguez Barrero, Mario Samuel;

Flórez Guzmán, Mario Heimer y Molina Ramírez, Adriana Rosa

empresa en los próximos tres años; se les preguntó a los encuestados, si tienen la intención de crear una empresa en los próximos 3 años. Este es un indicador relevante, pues quienes presentan la intención de hacerlo, es probable que intenten crear empresa, y por el contrario, quienes tienen la firme intención de no hacerlo, es muy probable que no lo hagan. En el programa de administración de empresas, se esperaría que una proporción significativa y mayoritaria de los estudiantes poseyera esta intención, si el programa está enfocado al emprendimiento. A continuación, se presentan los resultados de este índice, gráfico 4.

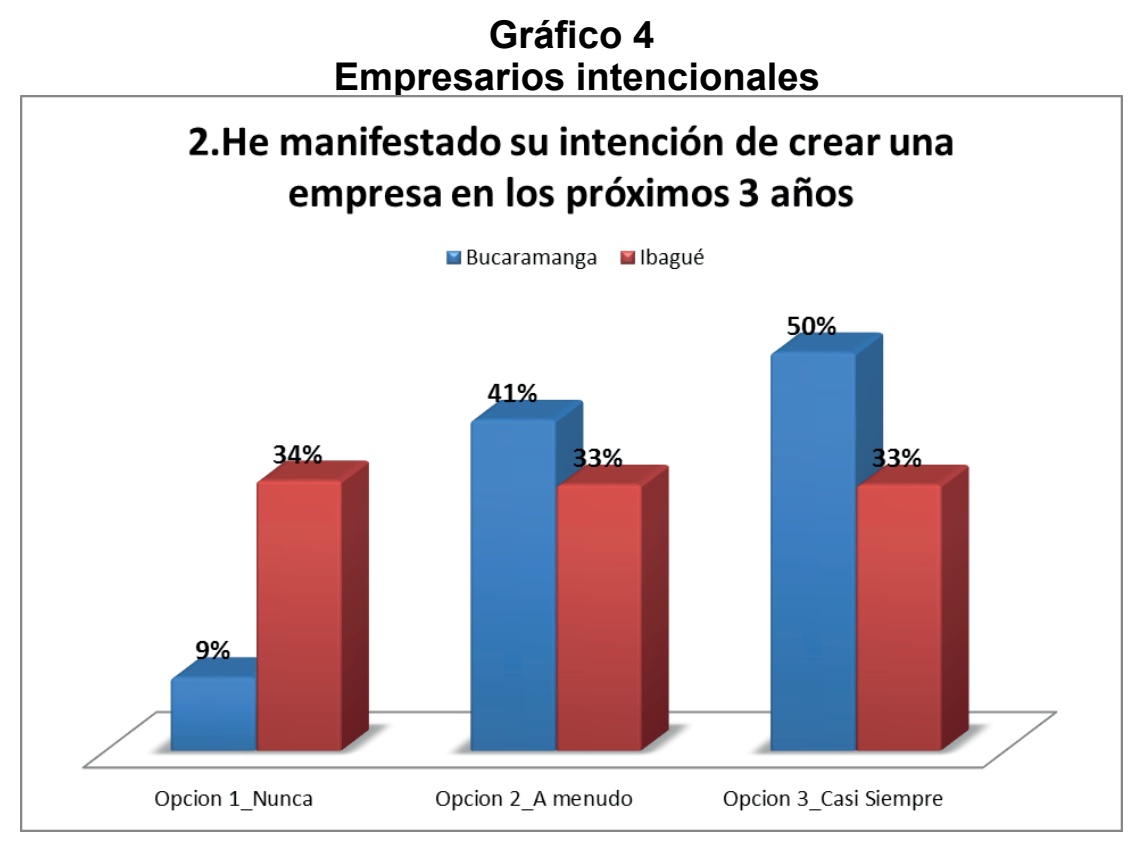

Fuente: elaboración propia

Estos resultados de intención, revelan que en la sede número 1 , un alto índice de estudiantes (34\%) manifiesta la intención de no crear empresa durante los próximos años, cifra que es llamativa para la universidad para que se motive a los estudiantes a crear empresa. En la sede número 2 , esta cifra fue del $9 \%$, lo cual es una cifra entendible, si no se tratara de un programa de administración, en el cual se esperarían cifras aún más bajas. Paralelamente, se observa que en la opción de casi siempre, que se refiere a los que tienen la intención de crear empresa, es del $50 \%$ en los estudiantes de la sede número 2 , y solo del $33 \%$ en la sede número 1 . 


\section{Conclusiones}

Como parte de las conclusiones del estudio, se puede considerar que el entorno de la sede número 2 (Bucaramanga) es más propicio para que los estudiantes perciban oportunidades para crear empresas que en la sede número 1 (Ibagué), pues pese a que entidades como el Banco Mundial y el Doing Bussiness ven a Ibagué como una ciudad con proyección, los jóvenes no lo perciben de la misma forma. Esto puede se puede deber a que cifras como el desempleo, subempleo, falta de oportunidades para estudiar y pobreza, se reflejan en mayor proporción en Ibagué, y esto afecta la percepción de oportunidades por parte de los jóvenes; también puede obedecer a aspectos culturales, pues es claro que hay culturas con mayor propensión al emprendimiento y otras con mayor aversión al riesgo.

De igual forma, se observó que existe una clara influencia del entorno social y económico de ciudad con respecto a las variables medidas, en dos ciudades que presentan procesos urbanos de crecimiento y desarrollo económico en los últimos años como es Bucaramanga e Ibagué. Sin embargo, de acuerdo a los resultados se puede concluir que el ambiente de emprendimiento se refleja con más propiedad en los estudiantes de la facultad de administración de la sede Bucaramanga.

Con respecto a la metodología GEM, se puede concluir que esta es una herramienta adecuada para la medición del emprendimiento en los estudiantes universitarios, aunque de ésta se puede derivar una herramienta específica para medir el emprendimiento universitario, el cual merece una medición especial, dado que es el emprendimiento con mayor tasa de efectividad, y con los resultados de estas mediciones, se pueden proponer políticas públicas y universitarias para promover el emprendimiento en los universitarios y apoyarlos para que emprendan.

La universidad estudiada bajo sus dos sedes, es una institución con un compromiso social, trayectoria y representatividad en el país, por lo cual, es importante que implemente estrategias para fomentar el emprendimiento con resultados. El emprendimiento puede ser transversal a sus ejes estratégicos de docencia, investigación y proyección social, lo cual facilita una mayor articulación con el mundo laboral, la empleabilidad, emprendimiento, empresarismo y la cohesión social. Este propósito será conmensurable, en la medida que los estudiantes tengan mayor propensión al emprendimiento, perciban oportunidades de emprendimiento, pierdan el miedo al fracaso, y salgan de la universidad con la firme intención de crear empresa, incluso que la creen durante su formación.

Por último, las políticas públicas deben favorecer más el emprendimiento, la Ley 1014 de 2006, de "Fomento a la cultura del emprendimiento", cumple más de una década y, aunque ha dado algunos resultados, es momento de evaluarlos y actualizar la normatividad, para que se implementen en las instituciones de educación estrategias efectivas que fomenten el emprendimiento.

\section{Referencias bibliográficas}

\footnotetext{
Alcaraz, Rafael (2011), El emprendedor de éxito. Cuarta Edición. McGraw Hill Interamericana Editores. Ciudad de México. México.
} 
Perfil emprendedor en estudiantes: divergencias entre dos universidades colombianas Rubio-Rodríguez, Gustavo Adolfo; Rodríguez Barrero, Mario Samuel;

Flórez Guzmán, Mario Heimer y Molina Ramírez, Adriana Rosa

Bargsted, Mariana (2013), El emprendimiento social desde una mirada psicosocial. Civilizar Ciencias Sociales y Humanas, Vol. 13, Núm. 25, pp 121-132.

Cantillon, Richard (1755). Essai sur la nature du commerce en general. London reprinted for Harvard University Press, Boston, G.H. Ellis, 1892.

Congreso de Colombia. (2006), Ley de fomento a la cultura del emprendimiento. [Ley 1014 de 2006]. Diario Oficial No. 46.164, Colombia.

Departamento Administrativo Nacional de Estadística DANE (2015), Pobreza monetaria y multidimensional-2015. Boletín Técnico. Bogotá, Colombia.

Duran, Sonia; Fuenmayor, Alejandro; Cárdenas, Sergio; Hernández, René (2016), Emprendimiento como proceso de responsabilidad social en instituciones de educación superior en Colombia y Venezuela. DesarroIlo Gerencial, Vol. 8, Núm. 2, pp 5875 .

Durán, Sonia; Parra, Margel; Márceles, Víctor (2015), Potenciación de habilidades para el desarrollo de emprendedores exitosos en el contexto universitario. Opción, Vol. 31, Núm. 77, pp 200-215.

Filion, Louis (2003), Réaliser son projet d'entreprise. 3éme édition. Collection Entrependre. Les éditions Transonctinental Inc. Québec. Canada.

Freire Gibb, Lucio; Nielsen Kristian (2011), Entrepreneurship within Urban and Rural Areas Individual Creativity and Social Network. DRUID Working Paper No. 11-01.

Gaete Quezada, Ricardo (2017), Responsabilidad social universitaria: una nueva mirada a la relación de la universidad con la sociedad desde la perspectiva de las partes interesadas. (Tesis doctoral). Universidad de Valladolid. España. Recuperado de: http://uvadoc. uva.es/bitstream/10324/923/1/TESIS148-120417.pdf

Global Entrepreneurship Monitor (2010), Global Report. Babson College \& Universidad del Desarrollo, Boston-Santiago de Chile.

Grebel, Thomas; Pyka, Andreas; Hanusch, Horst (2003), An Evolutionary Approach to Theory of Entrepreneurship. Industry and Innovation, Vol.10, Núm. 4, pp 415-493.

Hagen, Everett (1962), On the theory of Social Change. Homewood, IL: Dorsey.

Kelley, Donna; Singer, Slavica; Herrington, Mike (2016), Global Report 2015-2016. Report. Babson College \& Universidad del Desarrollo, Boston-Santiago de Chile.

Kruger, Maria (2004), Creativity in the entrepreneurship domain. Unpublished PhD thesis in Entrepreneurship, University of Pretoria. Pretoria, Gauteng. Sudáfrica.

McClelland, David (1960), The achieving society. Van Nostrand. Princeton, $\mathrm{NJ}$

O'Farrell, Patrick (1986). Entrepreneurship and regional development: Some conceptual issues. Regional Studies, Núm. 20, pp 565-574.

Ortiz Riagaa, María; Morales Rubiano, María (2011), Educación y Educadores - La extensión universitaria en América Latina: concepciones y tendencias. Revista Educación y educadores. Universidad de Nueva granada. pp 349-366.

Pico, Augusto; Del Rio, Jorge; Trujillo, Ricardo; Rodríguez, Carlos (2017), El emprendimiento como estrategia 
para el desarrollo humano y social. Saber, ciencia y libertad, Vol. 12, Núm. 1, pp 107-123.

Real Academia Española (1732). Diccionario de Autoridades - Tomo Tercero. $1^{\circ}$ Edición. Imprenta de la RAE. Madrid - España.

Ruiz Jiménez, Jeny; Cabeza Pulles, Dainelis; Briano Turrent, Guadalupe (2012), Universidad y emprendimiento: un caso de estudio en la Facultad de Ciencias Económicas y Empresariales. ReiDoCrea. Revista electrónica de investigación Docencia Creativa, Núm. 1, Universidad de Granada. pp 144-157.

Say, Jean Baptiste (1803), Traite d'economie politique. Kelley, New York.

Schumpeter, Joseph (1911), The Theory of Economic Development: An Inquiry into Profits, Capital, Credit, Interest and the Business Cycle. (R. Opie, Traductor), Cambridge: Harvard University Press (trabajo original publicado en 1911), 1934.

Selamé, Teresita (1999), Emprendimiento juvenil. Instituto Nacional de la Juventud. Santiago de Chile.
Torres, Claudia (2010). Consideraciones para la formación en emprendimiento: explorando nuevos ámbitos y posibilidades. Estudios gerenciales, Vol. 26, Núm. 117, pp 41-60.

Valera-Loza, Daniel; Vara-Horna, Arístides. (2007), El comportamiento emprendedor en las universidades. Universidad de San Martín de Porres. Lima. Perú.

Varela, Rodrigo; Gómez, Liyis; Vesga, Rafael; Pereira, Fernando (2014), Dinámica Empresarial Colombiana. Universidad Icesi, Universidad del Norte, Universidad de los Andes, Pontificia Universidad Javeriana. Cali. Colombia.

Varela, Rodrigo; Soler, Juan David (2012), GEM Caribbean 2011, Caribbean Regional Report. Universidad Icesi. Cali. Colombia.

Varela, Rodrigo; Soler, Juan David (2015), La tubería empresarial aplicada a algunos países del Caribe. Formación, innovación y éxito empresarial, Vol. 4, Universidad Icesi. Cali. Colombia.

- Esta obra está bajo una licencia de Creative Commons Reconocimiento-NoComercialCompartirlgual 3.0 Unported. http://creativecommons.org/licenses/by-nc-sa/3.0/deed.es_ES 